Вестник образования и развития науки Российской академии естественных наук, 2018, 22(2): 56-68 (C) С.И. Сороко, 2018

\title{
S.I. Soroko
}

\section{STUDY OF HUMAN ADAPTIVE POSSIBILITIES DURING PROLONGED STAY IN AUTONOMOUS LIFE SUPPORT SYSTEM}

Work is performed within the framework of the State job the FASO

(Reg. № AAAA-Ф18-118012290142-9)

Svyatoslav Soroko - Head of the Laboratory, Sechenov Institute of Evolutionary Physiology and Biochemistry of Russian Academy of Sciences, corresponding member of Russian Academy of Sciences, full member of Russian Academy of Natural Sciences, recipient of the State Prize of the USSR, Doctor of Medicine, professor, St. Petersburg; e-mail: soroko@iephb.ru.

We research specific characteristics of individual mechanisms of human adaptation to longterm isolation in a closed life support system during space flight simulation. The role of the plasticity of the nervous system in the restructuring of the brain bioelectrical activity, the quality of operator activity and psychological compatibility of crewmembers is considered. We also show the predictive value of the developed EEG-biofeedback technologies for the selection of people for working in small teams in the conditions of autonomous existence in objects equipped with a closed life support system.

Keywords: adaptation; modeling of space flights; social isolation; operator activity; brain; plasticity of nervous system; EEG-biofeedback technologies.

\author{
С.И. Сороко
}

\section{ИССЛЕДОВАНИЕ АДАПТИВНЫХ ВОЗМОЖНОСТЕЙ ЧЕЛОВЕКА В УСЛОВИЯХ ДЛИТЕЛЬНОГО ПРЕБЫВАНИЯ В АВТОНОМНОЙ СИСТЕМЕ ЖИЗНЕОБЕСПЕЧЕНИЯ}

Работа выполнена в рамках госзадания ФАНО (Рег. № АААА-Ф18-118012290142-9)

Святослав Иосифович Сороко - заведующий лабораторией Института эволюционной физиологии и биохимии им. И.М. Сеченова Российской академии наук, член-корреспондент РАН, действительный член РАЕН, лауреат Государственной премии СССР, доктор медицинских наук, профессор, г. СанктПетербург; e-mail: soroko@iephb.ru.

В статье рассматриваются особенности индивидуальных механизмов адаптации испытателей к длительной изоляции в замкнутой системе жизнеобеспечения при моделировании космических полётов. Рассмотрена роль пластичности нервной системы $в$ перестройках биоэлектрической активности мозга, качестве операторской деятельности, психологической совместимости членов экипажа. Показана прогностическая ценность разработанных НБОС-технологий для отбора лиц для работы в условиях автономного существования малочисленных коллективов в объектах с замкнутой системой жизнеобеспечения.

Ключевые слова: адаптация; моделирование космических полётов; социальная изоляция; операторская деятельность; мозг; пластичность нервной системы; НБОСтехнологии.

Успешным полётам человека в космос и созданию сложной технической и медико-биологической системы его жизне- обеспечения на космических станциях предшествовали многолетние исследования по адаптации и изучению резервных 
возможностей человека, поиску путей повышения его устойчивости в наземных экспериментах с моделированием факторов космического полёта и длительной изоляции в сурдокамерах, имитаторах космических кораблей, а также во время работы в суровых природных условиях (полярные и высокогорные станции, специальные экспедиции в отдаленные необитаемые районы и т.д.). При этом основное внимание уделялось влиянию на человека основных факторов космического полёта: невесомости, гиподинамии, сенсорной изоляции, изменению параметров внешней среды, различного рода перегрузкам.

Моделирование факторов невесомости осуществлялось с ограничением подвижности человека с помощью иммобилизации [13], длительного постельного режима [15], пребывания в ограниченном пространстве экспериментальных камер $[1 ; 5 ; 6 ; 17 ; 19]$, на специальных самолётах-лабораториях [4; 12]. Однако наиболее приближенными к космическим полетам следует считать наземные эксперименты с длительной изоляцией и максимальной имитацией условий полёта в специальных экспериментальных комплексах с автономной системой жизнеобеспечения [7].

Более полные сведения о результатах этих исследований можно найти в специальных изданиях $[2 ; 3 ; 7 ; 14]$.

Важное место в медико-биологических научных программах в автономных комплексах занимали психофизиологические исследования. Специальных исследований, направленных на объективное изучение нейрофизиологических механизмов и возможных дезадаптационных нарушений регулирующих функций мозга и его системной деятельности, в то время практически не проводилось в связи со сложностью съёма и телеметрической передачи многоканальной электроэнцефалограммы и вызванных потенциалов, отсутствием у экипажей специалистов, способных выполнять эти методики, отсутствием надёжной аппаратуры и самих подходов к изучению этих сложных функций мозга.

По инициативе директора Института медико-биологических проблем МЗ СССР академика О.Г. Газенко для проведения этих исследований в период 90- и 120суточного экспериментов в Наземном экспериментальном комплексе (НЭК) были привлечены сотрудники Лаборатории нейрокибернетики Института экспериментальной медицины АМН СССР, разработавшие оригинальные подходы, позволяющие в биотехнических системах с ЭЭГ-обратными связями изучать авторегуляционные свойства мозга и адаптивную пластичность нейродинамических процессов при воздействии на человека экстремальных факторов внешней среды [9]. Методы были апробированы на полярниках в условиях Антарктиды и оказались достаточно информативными [10].

Основной целью этих исследований являлось изучение роли пластичности нейродинамических процессов и индивидуальных типов механизмов саморегуляции мозга в механизмах адаптации человека к условиям изоляции в замкнутой системе жизнеобеспечения, психологической совместимости экипажа и устойчивости профессиональной деятельности.

К сожалению, результаты этих исследований были представлены только в виде научных отчётов в ИЭМБП МЗ СССР и в открытой печати не публиковались. В связи с тем, что в настоящее время проводятся Международные программы подготовки кандидатов к длительным межпланетным полётам к Марсу, нам представляется, что полученные нами данные в длительных экспериментах в Наземном экспериментальном комплексе могут представлять научный и практический интеpec.

Основными факторами, оказывающими отрицательное влияние на человека как в эксперименте, так и в реальных условиях обитания на подводных лодках, полярных станциях является длительная социальная изоляция небольшого по численности коллектива, сенсорная недостаточность, гиподинамия. Следовательно, многие факторы, особенно социальные, оказываются однотипными.

Краткая характеристика условий обитания в экспериментальном комплек- 
ce

Наземный экспериментальный комплекс (НЭК) представляет собой модель космического корабля. Это сложное уникальное инженерно-техническое сооружение с комплексом систем жизнеобеспечения рассчитано на длительный автономный режим работы с полной изоляцией от окружающей среды. НЭК предназначен для моделирования условий длительных космических полетов и поэтому имеет больший набор помещений и их объем, чем существующие в настоящее время космические аппараты.

Наши исследования в НЭКе проводились во время 90-суточного и 120-суточного экспериментов. Эксперименты отличались как по численности экипажа, так и по условиям их проведения. В 90-суточном эксперименте принимало участие три испытателя: К-ов, А-ин, М-ов. При проведении этого эксперимента испытатели точно знали о его продолжительности. Они были предупреждены, что замена членов экипажа в ходе эксперимента или досрочный выход из объекта практически исключены и могут быть разрешены только в случае серьезных заболеваний.

С целью изучения устойчивости испытателей к изменению параметров искусственной газовой атмосферы и возникающему при этом стрессу в ходе эксперимента были предусмотрены четыре внезапные аварийные ситуации (имитация неисправности системы жизнеобеспечения). Из них две аварийные ситуации были ложными, в которых специально изменялись только показания контрольно-измерительных приборов и увеличивалась температура и влажность, процентное соотношение газов в атмосфере было в нормe.

В 120-суточном эксперименте принимало участие шесть испытателей: П-ин, Ков, Т-ко, А-ин, Кл-ов, Г-ко. По условиям эксперимента была запланирована поочередная смена отдельных членов экипажа, причем трое из них должны были работать от начала до конца. Испытателям было неизвестно, кого и в какие сроки будут заменять. Таким образом, никто из членов экипажа фактически заранее не знал, сколько дней ему придется провести в НЭКе. На заключительном этапе эксперимента в объект на 4 дня вошло еще 3 человека из числа сотрудников, проводивших эксперимент.

Исследования проводились с 9.00 до 18.00 час. в одно и то же время для каждого испытателя. В течение экспериментов температура воздуха в жилом отсеке составляла плюс $20 \pm 5^{\circ} \mathrm{C}$, относительная влажность - 60-30\%. Концентрация кислорода равнялась 19,7-21\%, углекислого газа - 1,1-2\%. Содержание вредных примесей в окружающей атмосфере (окись углерода, аммиак, аминосоединения, органические примеси, сероводород и т.д.) не превышало уровней, регламентированных программой. При имитации аварийных ситуаций концентрация кислорода снижалась до $130 \pm 8$ мм рт. ст., а углекислого газа возрастала до 3,1-3,5\%, температура поддерживалась на уровне плюс $32 \pm 2^{\circ} \mathrm{C}$, влажность $-95 \%$.

Условия проведения эксперимента отличались довольно строгой социальной изоляцией. Выход на связь разрешался только в установленное время и регламентировался короткими служебными вопросами и ответами. При необходимости белее длинные инструкции или запросы передавались в письменном виде через специальную шлюзовую камеру с разрешения руководителя эксперимента. Во время медицинских исследований испытатели и экспериментаторы находились на связи в отведенное им время, но не более 60 мин., после чего ответственный дежурный центрального пульта связь выключал. Питание испытателей осуществлялось с использованием меню и продуктов, разработанных для реальных космических полётов.

Как было показано ранее [10], адаптивная пластичность нервной системы имеет выраженную индивидуальную зависимость и является основным свойством организма, определяющим характер и динамику развития адаптационного процесса в экстремальных условиях. В связи с этим мы считали целесообразным начать изложение результатов, полученных в длительных экспериментах с изоляцией 
малочисленных групп в замкнутой системе жизнеобеспечения, с оценки адаптивных свойств нервной системы испытателей.

Изучение адаптивных свойств нервной системы осуществлялось с помощью тестов, разработанных для полярников: методики произвольной регуляции параметров ЭЭГ и зрительно-моторного слежения за функцией собственной ЭЭГ и заданной кривой [10].

\section{0-суточный эксперимент}

И с п ы т а т е л ь К-ов (командир экипажа, один из инженеров-конструкторов НЭК). Испытатель К-ов в отличие от остальных участников 90-суточного эксперимента не является новичком в подобных исследованиях. В течение последних лет он неоднократно принимал участие в кратковременных (до 30 суток) экспериментах с изоляцией в НЭКе, связанных с отработкой систем жизнеобеспечения, моделированием аварийных ситуаций (пожара внутри объекта, повышенного радиационного фона, выхода из строя различных систем управления и т.д.). В данном эксперименте на испытателя К-ва была возложена обязанность командира экипажа. В связи с этим ответственность его в эксперименте была значительно выше, чем остальных участников.

Как в фоновых, так и в исследованиях, проведенных в различные сроки изоляции, у испытателя К-ва не было выявлено выраженной способности к произвольной регуляции амплитудно-частотных параметров ЭЭГ. Во время регуляции, направленной на увеличение или уменьшение амплитуды текущей ЭЭГ, изменение доминирующей частоты основных ритмов устойчивых направленных сдвигов указанных параметров не происходило. Некоторых успехов испытатель добивался в отдельных сеансах при выполнении пробы с «фокусировкой» и «расфокусировкой» (произвольная стабилизация или дестабилизация амплитудночастотных параметров ЭЭГ в условиях обратной связи по фигурам Лиссажу). При этом в ЭЭГ отмечалось выравнивание мощности отдельных составлявших частотного спектра и их стабилизация на оп- ределённом уровне (рис. 1). При анализе выраженности отдельных частот в ЭЭГ во время выполнения данной пробы обнаружены перестройки индексов. Оказалось, что если в состоянии покоя при открытых глазах индексы ритмов ЭЭГ составляли: дельта - 61,71\%, тета - 30,91\%, альфа $5,06 \%$, бета $-2,32 \%$, то при успешном выполнении пробы «фокусировка» эти соотношения изменились и стали равными: дельта - 42,93\%, тета - 39,05\%, альфа $12,9 \%$, бета $-5,1 \%$. Следует отметить, что поддерживать достигнутый сдвиг амплитудно-частотных параметров в условиях зрительной обратной связи испытатель Ков не мог. Уже через 30-60 с в электроэнцефалограмме появлялась дизритмия, частотный спектр становился неустойчивым, резко возрастал тета-индекс (до 66-70\%). Попытка повторной произвольной стабилизации параметров ЭЭГ испытателя, как правило, не удавалась. Внешне испытатель начинал проявлять повышенное беспокойство, раздражительность и самостоятельно либо прекращал выполнение задания, либо начинал относиться к нему формально. В последнем случае частотный спектр ЭЭГ возвращался к исходному уровню, бета-индекс снижался, альфаритм смещался в низкочастотную область $(7,8-8,9$ Гц).

Значительный рост выраженности тета-ритма, который отмечался во время выполнения тестов с произвольной регуляцией параметров ЭЭГ, по-видимому, является следствием выраженных отрицательных эмоций, возникающих у испытателя из-за невозможности достигнуть определённого успеха в выполнении задания [8; 11]. Выраженность эмоционального напряжения в процессе изоляции увеличивалась, однако наибольшей была па втором месяце эксперимента.

Исследования показали, что существенное влияние на выполнение тестов с произвольной регуляцией оказывал уровень мотивации испытателя, который был неодинаковым на разных стадиях адаптации. Мобилизующим фактором, значительно повышающим мотивационный уровень и ответственность испытателя при выполнении тех или иных медицин- 
ских исследований, оказались аварийные ситуации. В качестве примера можно привести результаты одного из исследований. В этот день исследование проводилось в условиях моделирования неполадок в системе жизнеобеспечения (ложная авария). Экипаж был занят поиском неисправностей, уровень нервно-эмоционального напряжения у всех испытателей был повышенным.

Однако точно по графику командир К-ов вышел на связь и доложил о готовности к проведению медицинского исследования. Испытатель был собран, точно выполнял все инструкции и показал хорошие результаты при выполнении тестов с произвольной регуляцией ЭЭГ. При выполнении пробы «фокусировка» перестройка мощностей отдельных частот достигла заданного уровня и стабильно удерживалась в течение всего сеанса, тета-индекс по сравнению с фоном был даже ниже. После прекращения пробы с регуляцией ЭЭГ отмечалось быстрое восстановление исходного профиля ЭЭГ. Произвольная регуляция в системе биоуправления, направленная на увеличение амплитуды отдельных частот ЭЭГ («рас- фокусировка»), испытателю К-ву не удавалась.

При попытках выполнить данную задачу происходило резкое увеличение в ЭЭГ выраженности низких частот (тета- и дельта-) во всем их диапазоне, снижение бета- и альфа-ритмов.

Во время опросов было установлено, что испытатель К-ов случаи успешной или неуспешной регуляции ЭЭГ с определенным внутренним состоянием не связывает. Однако при отключении зрительной обратной связи во время выполнения пробы и подмене ее сигналом, не связанным с текущей ЭЭГ (от генератора случайных напряжений), испытуемый быстро обнаруживает, что «сигнал не управляется».

Анализ амплитудно-частотных перестроек при выполнении операции слежения за огибающей собственной текущей ЭЭГ позволил исследовать индивидуально-типологические особенности пластичности авторегуляционных механизмов на различных стадиях формирования функциональной системы, обеспечивающей достижение полезного результата при выполнении операторских задач.

Ранее нами было показано, что каче-

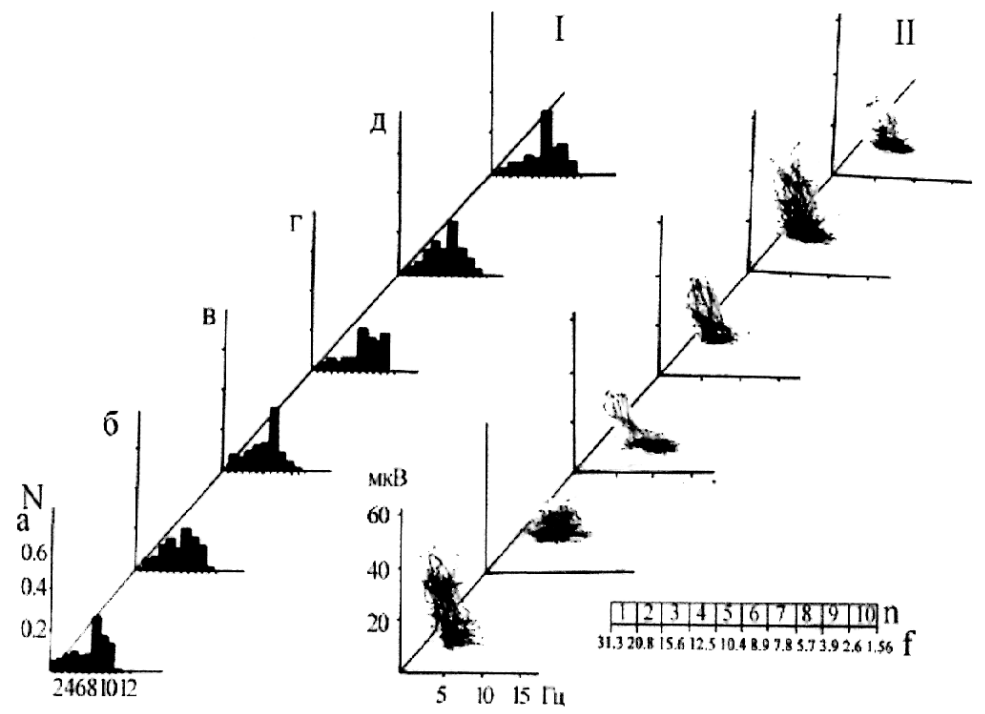

Рис. 1. Характер амплитудно-частотных перестроек ЭЭГ испытателя К-ва в различных функциональных режимах

I-гистограммы периодов ЭЭГ. По оси абсиисс - номер канала (частотный диапазон каждого канала приведен на шкале внизу рисунка), по оси ординат - число соответствующих периодов ЭЭГ за 2 мин. (частость). II - графики амплитудно-частотных зависимостей ЭЭГ. По оси абсиисс - частота в Ги, по оси ординат - амплитуда в мкВ. а - глаза открыты, б - глава закрыты, в-«фокусировка», 2- «расфокусировка», д-зрительно-моторное слежение за огибающей ЭЭГ, е-слежение за случайной кривой 
ство работы при слежении за огибающей текущей ЭЭГ зависит от возможности внутренней автоподстройки, направленной на стабилизацию амплитудно-частотных параметров [10].

Для выполнения операции зрительномоторного слежения в условиях НЭКа нами использовалась штатная система ближней стыковки космического корабля, состоящая из пульта управления и видеоконтрольного устройства. Для отслеживания заданного сигнала или огибающей амплитуды собственной ЭЭГ использовалась штатная ручка управления кораблём.

Исследования показали, что в отличие от тестов с произвольной регуляцией ЭЭГ, внутренняя автоподстройка амплитудно-частотных параметров с помощью авторегуляционных механизмов у испытателя К-ва в процессе слежения за ЭЭГ выражена несколько лучше. Однако длительной стабилизации параметров не наблюдается, качество слежения за ЭЭГ характеризуется неустойчивостью, вариабельностью параметров ЭЭГ. Значительная дисперсия показателей отмечается как в одном сеансе, так и в различных экспериментах. В дни с высокими показателями слежения сложность огибающей ЭЭГ уменьшается, однако качество слежения за случайной кривой, сложность которой не связана с текущим психофизиологическим состоянием, у испытателя К-ва всегда было выше. Это свидетельствует о преобладании у него внешнего, сенсомоторного, контура регулирования в структурной схеме зрительно-моторного слежения и недостаточной пластичности механизмов внутренней саморегуляции [10].

Повышенный уровень нервно-эмоционального напряжения, сопровождающийся резким увеличением в ЭЭГ тета- и дельта-ритмов, которое всегда отмечалось у К-ва в ходе сеанса связи, неустойчивость и относительная слабость тормозных механизмов часто приводили к возникновению «отказов деятельности». В результате испытатель после значительного числа ошибок либо полностью прекращал слежение, либо начинал отслеживать только отдельные высокоамплитудные колебания.
И с п ы т а т е л ь А-ин (бортинженер). В эксперименте с изоляцией участвует впервые. Фоновые исследования перед началом эксперимента показали, что исходная ЭЭГ испытателя А-на характеризуется значительной стабильностью частотного спектра, который трудно поддается произвольной регуляции. Незначительное изменение параметров при выполнении пробы «фокусировка» отмечалось в первые дни эксперимента. С увеличением срока пребывания в НЭКе даже эта возможность произвольной регуляции утратилась. Пробы с «расфокусировкой», направленные на увеличение амплитуды ЭЭГ или расширение диапазона частотного спектра испытатель выполнять не мог (рис. 2). При выполнении проб с произвольной регуляцией ЭЭГ отмечалось значительное увеличение медленных частот в диапазоне 2,6-5,7 Гц и снижение частоты в диапазоне 8,9-31,3 Гц, т.е. в области альфа- и бета-ритмов. Если задача, направленная на выравнивание мощности отдельных составляющих, сопровождалась незначительной перестройкой исходного спектра, особенно в области альфаритма, то при попытках выполнить тест «расфокусировки» происходил заметный сдвиг частотного спектра в сторону более высоких частот, особенно увеличивалось число периодов, соответствующих бетаритму. В процессе длительной изоляции в НЭКе у испытателя А-на произошло резкое смещение частотного спектра в сторону медленных ритмов. Этот сдвиг частотного спектра ЭЭГ оказался довольно устойчивым и сохранялся при выполнении тестов с переработкой информации.

У испытателя А-на на втором месяце эксперимента отмечено появление депрессивного состояния, которое проявлялось в общей заторможенности, безразличии, вялости, потере аппетита, собственной неудовлетворенности. В ЭЭГ отмечалось значительное число медленных составляющих, пробы с переработкой информации вызывали не депрессию, а экзальтацию альфа-ритма в его низкочастотной полосе $(7,8-8,9$ Гц). Анализ частотного спектра ЭЭГ при выполнении различных функциональных проб и в пе- 
риоды последействия выявил у А-на значительную инерционность процессов торможения и ее увеличение в ходе эксперимента.

В исследованиях со зрительномоторным слежением за огибающей амплитуды собственной ЭЭГ внутренней самоподстройки параметров ЭЭГ, направленной на упрощение отслеживаемой функции и минимизацию ошибок, у испытателя не обнаружено. Качество слежения
А-на при слежении за огибающей ЭЭГ и за заданной кривой, отличалось неустойчивостью (рис. 3 ).

И с п ы т а т е л ь М-ов (врач экипажа). В эксперименте с длительной изоляцией участвует второй раз. В исследованиях с ЭЭГ-обратными связями у испытателя М-ва обнаружена выраженная пластичность нейродинамических процессов. Свойства высокой пластичности проявились как в тестах с произвольной регуля-

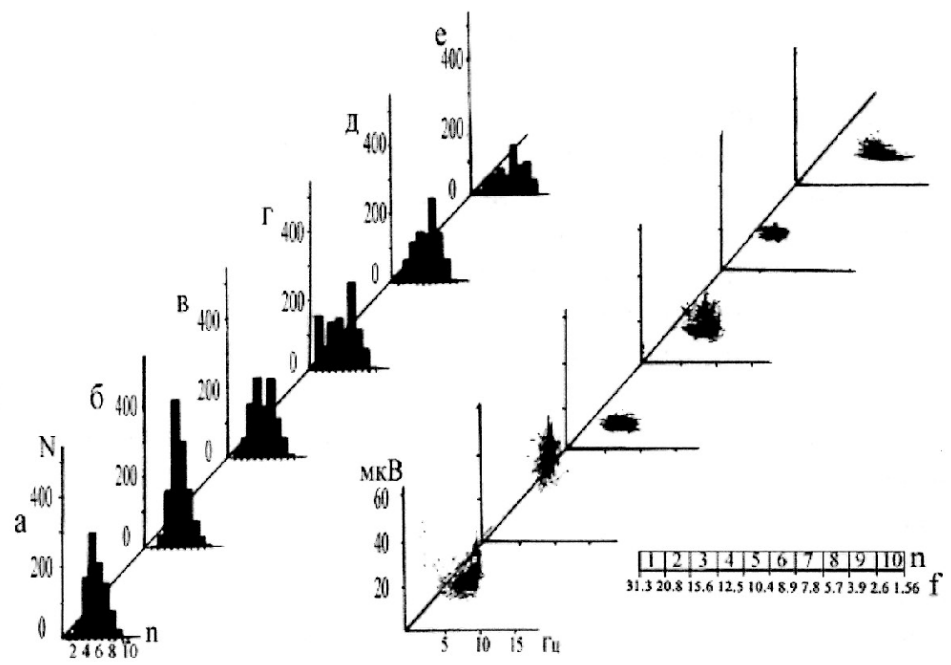

Рис. 2. Характер амплитудно-частотных перестроек ЭЭГ у испытателя А-на в различных функциональных режимах

Обозначения те же, что и на рис. 1

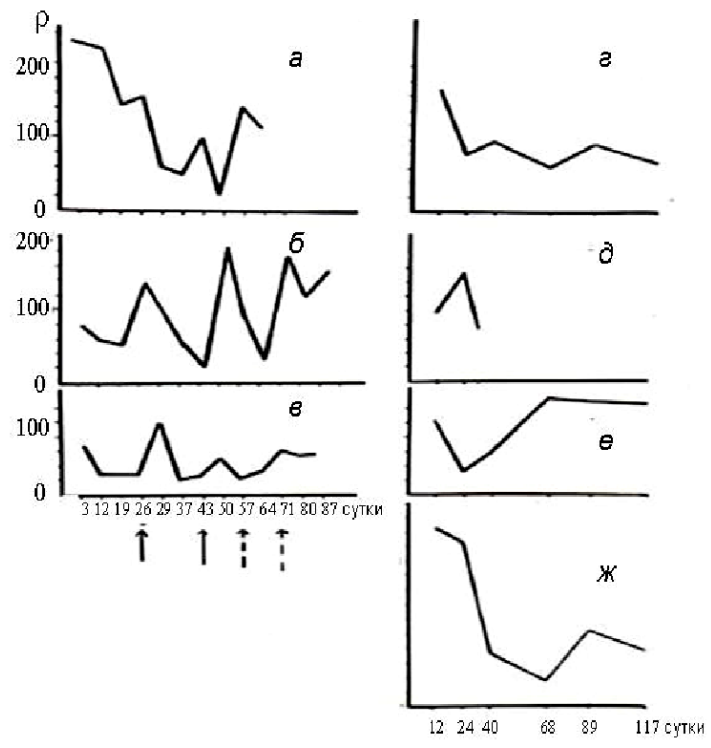

Рис. 3. Динамика качества работы испытателей в 90- $(a, 6$, , в) и 120-суточном $(2, \partial, e, ж)$ экспериментах при выполнении зрительно-моторного слежения

На графиках: по оси абсиисс - разные периода изолящии (в сутках), по оси ординат - величина ошибки ( $\rho)$. Сплошная стрелка - аварийная ситуация, прерывистая - ложная аварийная ситуачия, а, г-испытатель К-ов; б, д-испытатель А-ин; в-испытатель М-ов;

$$
\text { e-испытатель П-ин; ж-испытатель Т-ко }
$$


цией амплитудно-частотных параметров ЭЭГ, так и при выполнении зрительномоторного слежения за огибающей собственной ЭЭГ.

Исследования показали, что уже со второго опыта испытатель М-ов начал успешно выполнять произвольную регуляцию, направленную на снижение или увеличение амплитуды ЭЭГ и стабилизацию частотного спектра, и поддерживать достигнутые сдвиги в течение 3-х минут. Перестройка амплитудно-частотных параметров ЭЭГ в соответствии с задачей произвольной регуляции наступала практически сразу после подачи команды.

Поскольку исходная ЭЭГ М-ва отличалась выраженным альфа-ритмом, проба «фокусировка» осуществлялась преимущественно за счет перестроек удельного веса отдельных диапазонов внутри этого ритма, при незначительном изменении бета-ритма (рис. 4). Во время «фокусировки» несколько увеличивается число периодов, соответствующих бета-ритму в диапазоне 15,6-20,8 Гц, незначительно уменьшается диапазон альфа-ритма 12,510,4 Гц и существенно падает выраженность альфа-ритма в полосе 8,9-10,4 Гц и 7,8-8,9 Гц (5 и 6 канал на гистограмме), отмечается увеличение тета-ритма в диапазоне 5,7-7,8 Гц.

Выраженность частот, лежащих в полосе 1,56-2,6 Гц, во время выполнения данной пробы практически не меняется.
Исходное значение индексов отдельных частот, которое у М-ва выражалось значениями: дельта $-2,95 \%$, тета - 12,32\%, альфа $-76,22 \%$, бета $-8,52 \%$, во время выполнения пробы «фокусировка» изменялось следующим образом: дельта $20,17 \%$, тета - 22,71\%, альфа - 47,84\%, бета $-9,28 \%$.

Регуляция ЭЭГ, направленная на увеличение амплитуды, происходила за счет роста удельного веса высокочастотного диапазона тета-ритма $(5,7-7,8$ Гц $)$ и уменьшения выраженности альфа-ритма во всем его диапазоне. Однако поддержание указанного сдвига давалось с трудом и, как правило, непроизвольно переходило к противоположному сдвигу, то есть к режиму, соответствующему тесту «фокусировка».

Необходимо отметить, что все виды переработки информации осуществлялись у М-ва на фоне довольно выраженного альфа-ритма. Качество произвольной регуляции сохранилось в течение всего эксперимента, хотя с увеличением срока изоляции выполнение указанных проб давалось с большим напряжением нервной системы, о чём свидетельствует постепенный рост от опыта к опыту тета-ритма, сначала в области 5,7-7,8 Гц, а затем в области 3,9-5,7 Гц. В последние дни пребывания в НЭКе отмечается некоторое увеличение дельта-ритма. После выхода из объекта выполнение проб с произвольной

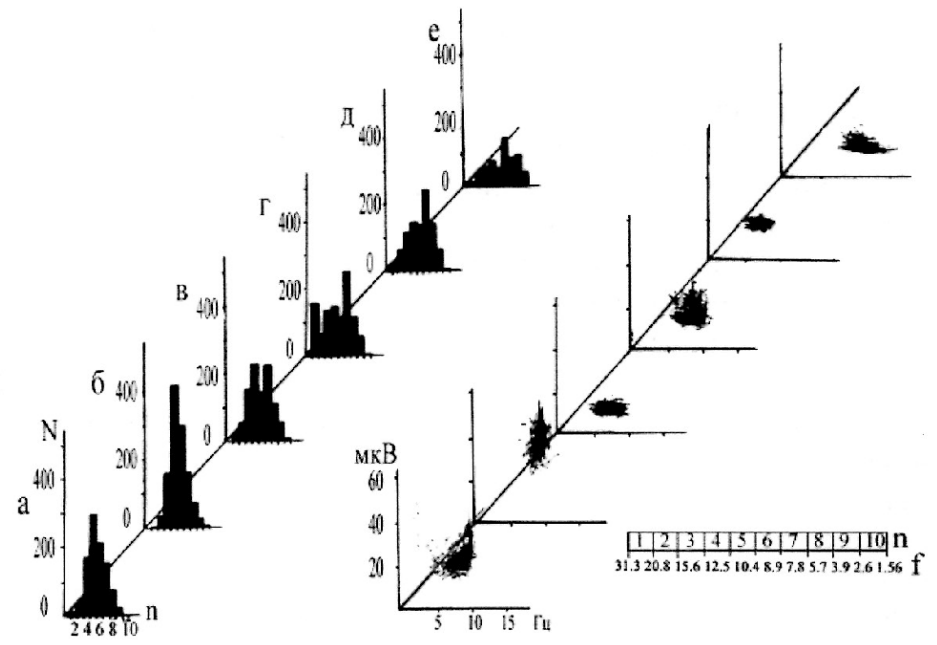

Рис. 4. Характер амплитудно-частотных перестроек ЭЭГ у испытателя М-ва в различных функциональных режимах Обозначения те же, что и на рис. 1 
регуляцией происходило без особого увеличения тета-ритма, которое отмечалось во второй половине эксперимента.

Следует отметить высокую подвижность и пластичность основных нервных процессов у испытателя М-ва. Восстановление исходных характеристик ЭЭГ в последействии происходит у него практически мгновенно во всех пробах. В конце исследования М-ов мог выполнять произвольную регуляцию параметров ЭЭГ и в условиях выключенной обратной связи. Однако, в этом случае очень быстро происходило «ускользание» достигнутого сдвига, т.е. возвращение параметров ЭЭГ к исходному уровню.

Испытатель М-ов показал высокое качество слежения на протяжении всех трех месяцев эксперимента (рис. 3, в). При анализе результатов было обнаружено, что точность работы при слежении за огибающей ЭЭГ у него почти всегда была выше, чем при отслеживании заданной случайной кривой. На рис. 3 в видно, что показатель точности слежения р имеет меньший разброс от опыта к опыту. При слежении за случайной кривой показатель имеет большую вариабельность.

Анализ амплитудно-частотных параметров ЭЭГ показал, что в процессе слежения за огибающей электроэнцефалограммы происходит выраженная стабилизация, как частоты, так и амплитуды. Перестройки ЭЭГ идут в направлении упрощения отслеживаемой функции, т.е. снижения ее сложности. Это позволяет испытателю не только достаточно точно отслеживать все колебания огибающей, но и увеличивает вероятность прогнозирования. Адаптивная самоподстройка ЭЭГ, отсутствие повышенного эмоционального напряжения во время выполнения операции слежения, быстрая реакция позволили испытателю отслеживать даже высокочастотные колебания.

\section{0-суточный эксперимент}

И с п ы т а т е л ь П-ин (командир и врач экипажа). В эксперименте с изоляцией участвует впервые. На протяжении всего эксперимента с длительной изоляцией выраженной способности к произвольной регуляции параметров ЭЭГ у ис- пытателя не обнаружено. При попытке произвольной регуляции в ЭЭГ П-на отмечается незначительное уменьшение амплитуды, однако частотный спектр практически не отличается от частотного спектра исходной ЭЭГ. На рис. 5 представлены графики амплитудно-частотных зависимостей ЭЭГ в различных функциональных режимах, полученные на 40 сутки. Каждая точка на графике отражает среднее значение интеграла амплитуды (ось ординат) и частоты (ось абсцисс) за каждые последовательные 5 с. Видно, что в режиме «фокусировка» существенного уменьшения дисперсии показателей не отмечается.

Однако в режиме «пседофокусировка», когда испытуемому предлагалось стабилизировать параметры ЭЭГ при подмене собственной функции ЭЭГ искусственной кривой (фактическое выключение обратной связи), дисперсия амплитуды и частоты ЭЭГ существенно возрастает. Это указывает на то, что некоторые элементы произвольной регуляции собственной ЭЭГ у испытателя имеются. Незначительно выраженные способности к регуляции параметров ЭЭГ отмечались у испытателя на 89, 117 сутки и через 4 суток после выхода из объекта.

В этом случае в частотном спектре ЭЭГ отмечается выравнивание составляющих бета-ритма, снижается альфаритм. Доминирующая частота альфа-ритма 7,8-8,9 Гц уменьшается и увеличивается число периодов ЭЭГ, соответствующих диапазону 8,9-10,4 Гц и 10,4-12,5 Гц. Происходит увеличение интенсивности тета-ритма в диапазоне 5,7-7,8 Гц и уменьшается в полосе 3,9-5,7 Гц. Возрастает число периодов, соответствующих частоте 3,13-3,9 Гц по сравнению с диапазоном 2,6-3,73 Гц.

Исследования показали, что испытатель в отдельных случаях способен осуществлять произвольную регуляцию параметров ЭЭГ, однако пределы и устойчивость этой регуляции невелики. В повторных исследованиях существенного повышения этих возможностей за счёт обучения не отмечалось. П-ин не обнаружил определённой связи внутреннего со- 


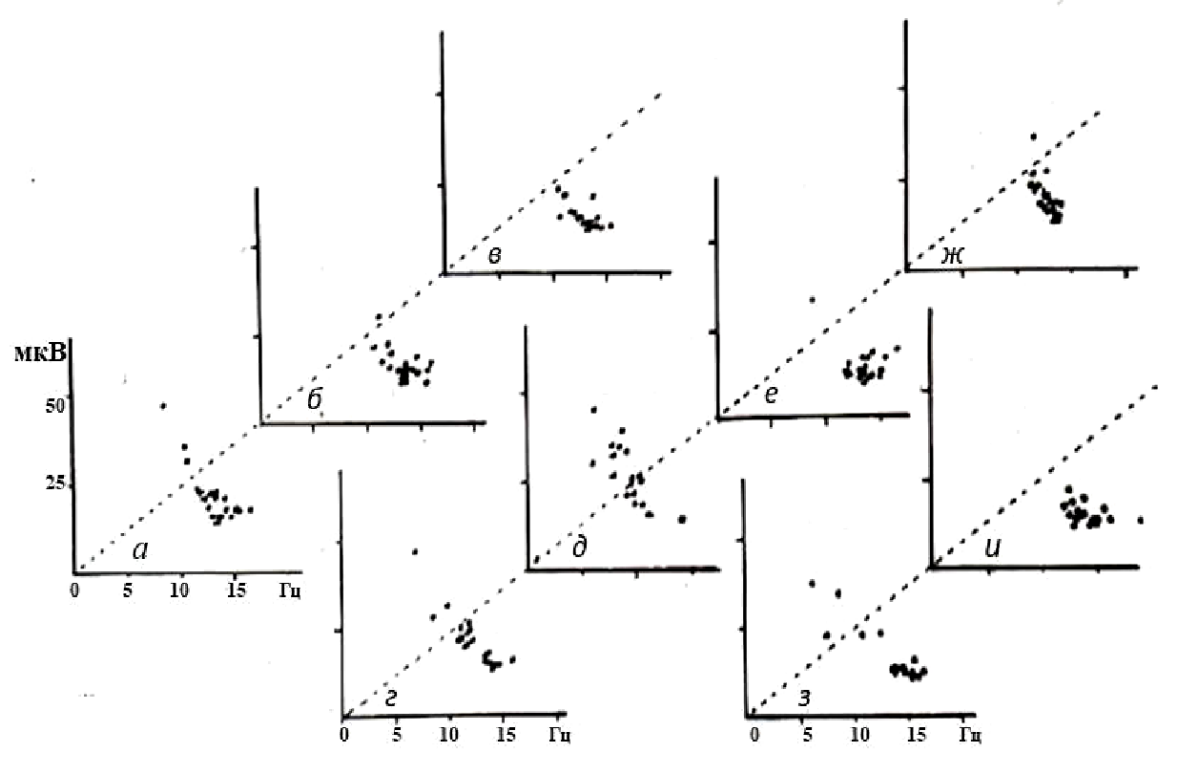

Рис. 5. Амплитудно-частотные зависимости ЭЭГ испытателя П-на в различных функциональных режимах

По оси абсиисс - среднее значение частоты ЭЭГ в Ги за каждые последовательные 5 с; по оси ординат - среднее значение интеграла амплитуды ЭЭГ за то же время в мкВ. а-глаза открыты, б - глаза закрыты, в-режим «фокусировка», г-последействие "фокусировки», д-режим «псевдофокусировка», е-слежение за огибающей ЭЭГ, ж-последействие после слежения за огибающей ЭЭГ, з - слежение за случайной кривой, и-последействие после слежения за случайной кривой

стояния с качеством регуляции.

Динамика слежения испытателя П-на имеет 3 фазы. В начале 120-суточного эксперимента (12 сутки) качество слежения за огибающей ЭЭГ, как и за случайной кривой, было низким (рис. $3, e$ ). Однако уже в этом эксперименте испытатель обнаружил способность стабилизировать амплитудно-частотные параметры ЭЭГ, при этом у испытателя доминирует бетаритм. На протяжении последующих 35-40 суток сложность ЭЭГ практически не меняется, однако уровень ошибок уменьшается, что свидетельствует о том, что П-ин добивается высокой точности не только за счет упрощения огибающей ЭЭГ, но и за счет ручной коррекции с помощью «ручки управления». Начиная со второго месяца изоляции, качество ухудшается, падает бета-индекс, нарастает тета-индекс, сложность огибающей ЭЭГ возрастает.

Аналог слежения за случайной и регулярной кривой дает ту же динамику во времени. Сравнение же результатов П-на с результатами других испытателей говорит о невысокой роли моторного компонента в процессе слежения. К концу экс- перимента (117 сутки) точность повышается, достигая величины, наблюдаемой на 26 сутки четырехмесячного эксперимента. В целом, при слежении за огибающей ЭЭГ испытатель добивался лучших результатов, чем при слежении за случайной кривой. Зависимости между качеством слежения и количеством членов экипажа в НЭКе, определяющим концентрации $\mathrm{CO}_{2}$, не установлено.

И с п ы т а т е л ь Т-ко (инженер). В подобном эксперименте участвует впервые. Исследования показали, что испытатель Т-ко обладает хорошей способностью к произвольной регуляции параметров собственной ЭЭГ в условиях обратной связи, информирущей о результатах деятельности. На рис. 6, II видно, что в режиме «фокусировка» происходит увеличение бета-ритма в диапазонах 15,620,8 Гц и 20,8-31,3 Гц, снижение альфаритма, особенно в диапазоне 8,9-10,4 Гц, увеличение тета-ритма. Дисперсия амплитуды и частоты снижается (рис. 6, I, в). После прекращения «фокусировки» существенно возрастает разброс показателей, увеличивается амплитуда ЭЭГ (рис. 6, I, 
2). На гистограмме периодов ЭЭГ (рис. 6, II, г) отмечается уменьшение бета-ритма во всем его диапазоне (каналы I, 2, 3), снижение выраженности альфа-ритма в полосе 10,4-12,3 Гц (канал 4). Одновременно происходит рост тета- и дельтаритма, что указывает на высокое нервноэмоциональное напряжение испытателя, которое отмечается в первые 3-5 минут после режима с произвольной регуляцией ЭЭГ. При этом амплитуда ЭЭГ варьировала в больших пределах, менялась частота ЭЭГ. В режиме «псевдофокусировка» отмечается рост тета-индекса, что очевидно, связано с отрицательными эмоциями, возникающими у испытателя вследствие несоответствия между его усилиями и результатами.

В динамике точности слежения за огибающей собственной ЭЭГ также можно выделить 3 периода - начальный, длящийся около 35 суток и характеризующийся значительными ошибками (рис. 3, ж).

На втором этапе уровень ошибок падает - происходит это в основном за счёт умеренно выраженной стабилизации ам- плитудных параметров ЭЭГ. Начиная примерно с 60-65 суток (счёт ведётся от начала пребывания в НЭКе Т-ко) и до конца эксперимента стабилизация амплитуды автоподстраивающейся ЭЭГ имеет неустойчивый характер, вследствие чего уровень ошибок вновь возрастает, не достигая значений, полученных в начале эксперимента. Слежение испытателя Т-ко сопровождается повышением в ЭЭГ бетаиндекса. Чёткой динамики в качестве слежения за случайной кривой проследить не удаётся, однако к концу эксперимента уровень ошибок повышается, а на 4 сутки после выхода из объекта снижается до исходного уровня (3 сутки изоляции или 40 сутки от начала четырёхмесячного эксперимента).

Заключение. Оценка качества работы оператора при слежении за заданной кривой имеет важное практическое значение. Однако одной из основных целей нашей работы являлось использование этой методики для изучения авторегуляционных способностей мозга. Качество слежения за функцией собственной ЭЭГ главным образом определяется авторегуляционными

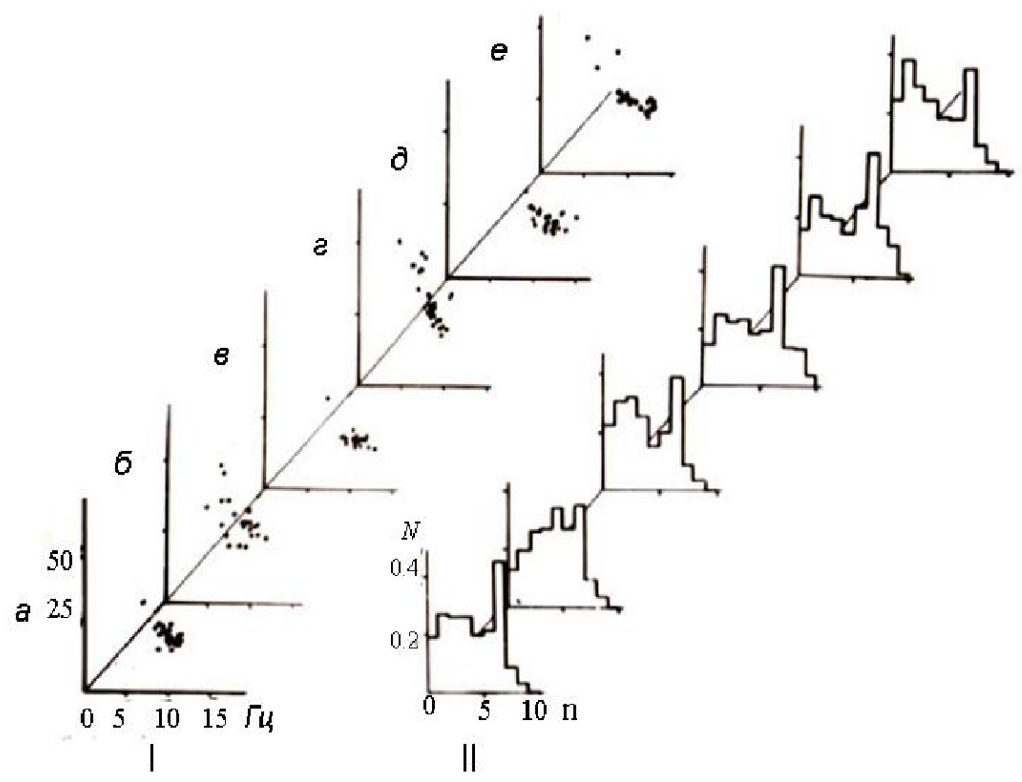

Рис. 6. Амплитудно-частотные зависимости (I) и гистограммы ЭЭГ (II) испытателя Т-ко в различных режимах на третьи сутки изоляции в НЭКе.

a-глаза открыты, б - глава закрыты, в- «фокусировка», г-последствие, д-слежение за огибающей ЭЭГ, е-слежение за случайной кривой. На гистограммах: по оси абсцисс - номера каналов, по оси ординат - частоты. Полосы частот каждого канала указаны в таблице.

Остальные обозначения те же, что и на рис. 4 
свойствами мозга оператора.

Так, активная «внутренняя самоподстройка» в трёхмесячном эксперименте отмечалась только у испытателя М-ва, у испытателей К-ва и А-на этот феномен не обнаружен. Вследствие этого качество слежения за ЭЭГ у испытателя М-ва было выше, чем при слежении за заданной кривой. Это ещё раз подтверждает тот факт, что М-ов обладает более высокими адаптивными свойствами ВНД, уравновешенностью основных нервных процессов и большей их подвижностью.

В общей динамике качества слежения в системе с ЭЭГ-обратными связями в процессе трёхмесячной изоляции в НЭКе можно выделить три периода: первоначальный период высоких шибок, период постепенного улучшения операторской деятельности и, период ухудшения показателей точности зрительно-моторного слежения к концу эксперимента. Первый период занимал время от 15 дней у М-ва до 40 дней у К-ва. Длительность второго периода составляла для К-ва 35 дней, дня испытателя М-ва - 60 дней.

Выраженность изменений в ЭЭГ и динамика точности работы в системе зрительно-моторного слежения в данном эксперименте и её сопоставление с результатами других авторов свидетельствует о большой роли установки на продолжительность эксперимента.

Указанные фазы работоспособности были выявлены нами у полярников антарктической станции [10], отмечались они и в годовом наземном медико-биологическом эксперименте [6]. Однако периоды были более растянуты во времени. Некоторая зависимость выраженности изменений в функциональном состоянии организма в условиях изоляции от установки на время эксперимента отмечена [18]. В 120-суточном эксперименте автоподстройка ЭЭГ в процессе слежения за огибающей отмечена практически у всех испытателей. Однако наиболее выраженной она оказалась у П-на (в первые 50 суток эксперимента) и А-на, наименее - у Ква. Лучших показателей при слежении за огибающей ЭЭГ по сравнению с испытателем Т-ко он достиг за счёт более коор- динированных движений «ручкой» управления. Наилучшие результаты, достигнутые П-ым в процессе слежения за регулярной кривой, подтверждают этот вывод.

120-суточный эксперимент отличался тем, что испытателям не были известны сроки выхода из объекта. Кривые качества работы К-ва и А-на не имеют трёхфазной формы, характерной для 90-суточного эксперимента. У испытателя К-ва нет выраженных стадий перестроек ЭЗГ, характерных для других испытателей. На основании этого можно предположить, что Ков в течение всего эксперимента не был уверен в том, что он не будет заменён другим испытателем.

В динамике качества деятельности Пна и Т-ко эти три фазы выявляются довольно отчётливо. Можно полагать, что П-ин, как командир и как единственный врач среди испытателей, рассчитывал проработать в НЭКе в течение всего эксперимента. Т-ко включился в работу на 38 сутки и, как выяснилось позже, располагал информацией о сроках своего пребывания в условиях изоляции.

120-суточный эксперимент подтвердил, что динамика процесса адаптации с изоляцией в модели космического корабля имеет определённую закономерность и зависит от установки испытателя на срок окончания эксперимента. Если продолжительность эксперимента неизвестна, адаптационные перестройки происходят при более высоком напряжении основных нервных процессов.

Таким образом, проведённые исследования показали, что устойчивость человека к длительной социальной изоляции и сенсорной недостаточности во многом определяется адаптивной пластичностью и устойчивостью центральных механизмов, осуществляющих регуляцию мозгового гомеостаза. Оценка этих свойств нервной системы человека может производиться с помощью системных методик с биологическими обратными связями. Полученные данные показали, что выраженность адаптивных свойств может являться одним из возможных прогностических критериев при профессиональном отборе лиц, направленных для работы в малые 
изолированные коллективы, а также для отбора операторов систем управления.

\section{ЛИТЕРАТУРА}

1. Агаджанян Н.А., Бизин Ю.П., Доронин Г.П., Ильин Е.А., Кузнецов А.Г., Езенчук Н.И. О влиянии на организм человека длительного пребывания в замкнутой камере малого объёма // Проблемы космической биологии. 1965. Т. 4. С. 3143.

2. Газенко О.Г. (ред.). Человек в длительном космическом полете. М.: Мир, $1974.360 \mathrm{c}$.

3. Газенко О.Г., Кальвин М. Основы космической биологии и медицины. М.: Наука, 1975. 560 с.

4. Китаев-Смык Л.А. Реакции людей в невесомости // Проблемы космической биологии. М.: Наука, 1964. Т. 3. С. 159168.

5. Новиков В.С., Бедненко В.С., Ушаков И.Б. Основы космической физиологии // Физиология лётного труда. СПб.: Наука, 1997. C. 343-395.

6. Парин В.В., Космолинский Ф.П., Душков Б.А. Космическая биология и медицина. М.: Просвещение, 1970. 223 с.

7. Сенкевич Ю.А., Пишак В.Б., Николаев С.О., Круговых В.В., Смирнова Т.А. Аналитический обзор зарубежной литературы по проблемам космической биологии и медицины за 1978 г. // Бюллетень космической биологии и медицины. 1979. T. 3. № 4. С. 3-40.

8. Симонов П.В. Высшая нервная деятельность человека. Мотивационно-эмоциональные аспекты. М.: Наука, 1975. 173 c.

9. Сороко С.И., Суворов Н.Б., Бекшаев C.C. Произвольный контроль уровня биоэлектрической активности мозга как метод изучения авторегуляционных свойств центральной нервной системы // Адаптивная саморегуляция функций. М.: Медици- на, 1977. С. 206-248.

10. Сороко С.И., Бекшаев С.С., Сидоров Ю.А. Основные типы механизмов саморегуляции мозга. Л.: Наука, 1990. 205 с.

11. Судаков К.В. Системные механизмы эмоционального стресса. М.: Медицина, $1981.232 \mathrm{c}$.

12. Хрунов Е.В., Хачатурьяни Л.С., Попов В.А., Иванов Е.А. Человек-оператор в космическом полете. М.: Машиностроение, 1974. $400 \mathrm{c}$.

13. Deitrick J.E., Whedon G.D., Shorr E. Effects of immobilization upon various metabolic and physiologic functions of normal man // Amer. J. Med. 1948. V. 4. P. 3-36.

14. Fraser T.H. Leisure a reaction in long duration space missions // Hum. Factors. 1968. V. 10. P. 483-488.

15. Greenleaf J.E., Bernauer E.M., Juhos L.T., Young H.L., Morse J.T., Staley R.W. Effects of exercise on fluid exchange and body composition in man during 14-day bed rest // J. Appl. Physiol. 1977.V. 43. № 1. P. $126-132$.

16. Hargreaves J.S., Robertson W.G., Ulvedal F., Zeft H.J., Welch B.E. Study of man during a 56-day exposure to a helimoxigen atmosphere at $258 \mathrm{~mm} \mathrm{Hg}$ total pressure. Introduction and general design // Aerospace Med. 1966. V. 37. P. 552-564.

17. Rockwell D.A., Hodgson M.G., Beljan J.R., Winget C.M. Psychologic and psychophysiological response to 105-days social isolation // Aviat. Space environ. Med. 1976. V. 47. № 10. P. 1087-1093.

18. Suedfeld $P$. Change in intellectual performers and in susceptibility to influence // Sensory Deprivation: Fifteen Years of Research / Ed. by J.P. Zubek. N.Y., 1969. P. 126-166.

19. Taliaferro E.H., Seagman J.S. Medical and behavioral aspects of long-term manned space cabin test // San Francisco: Aerospace Med. Assoc., 1969. P. 63-66. 